\title{
Clinicopathologic and Prognostic Value of Serum Carbohydrate Antigen 19-9 in Gastric Cancer: A Meta-Analysis
}

\author{
Yong-xi Song, Xuan-zhang Huang, Peng Gao, Jing-xu Sun, Xiao-wan Chen, Yu-chong Yang, \\ Cong Zhang, Hong-peng Liu, Hong-chi Wang, and Zhen-ning Wang
}

Department of Surgical Oncology and General Surgery, First Hospital of China Medical University, 155 North Nanjing Street, Heping District, Shenyang 110001, China

Correspondence should be addressed to Zhen-ning Wang; josieon826@sina.cn

Received 20 July 2015; Accepted 29 September 2015

Academic Editor: Ralf Lichtinghagen

Copyright (C) 2015 Yong-xi Song et al. This is an open access article distributed under the Creative Commons Attribution License, which permits unrestricted use, distribution, and reproduction in any medium, provided the original work is properly cited.

Background. The clinical value of carbohydrate antigen (CA) 19-9 in gastric cancer is controversial. We evaluated the clinicopathologic and prognostic value of CA 19-9 in gastric cancer. Methods. A literature search was conducted in PubMed and Embase databases. Odds ratios (ORs), risk ratios (RR), hazard ratios (HRs), and 95\% confidence intervals (CIs) were used as effect measures. Results. Thirty-eight studies were included. Results showed that there were significant differences in the incidence of high CA 19-9 levels between stages III/IV and I/II groups $(\mathrm{OR}=3.36$; $95 \% \mathrm{CI}=2.34-4.84)$, the $\mathrm{pT} 3 / \mathrm{T} 4$ and pT1/T2 groups $(\mathrm{OR}=2.40$; $95 \% \mathrm{CI}=1.60-3.59)$, the lymph node-positive and node-negative groups ( $\mathrm{OR}=2.91 ; 95 \% \mathrm{CI}=2.21-3.84)$, the metastasis-positive and metastasis-negative groups $(\mathrm{OR}=2.76 ; 95 \% \mathrm{CI}=1.12-6.82)$, and vessel invasion-positive and invasion-negative groups $(\mathrm{OR}=$ 1.66 ; $95 \% \mathrm{CI}=1.11-2.48)$. Moreover, CA $19-9$ was significantly associated with poor overall survival $(\mathrm{HR}=1.83 ; 95 \% \mathrm{CI}=1.56-2.15)$, disease-free survival $(\mathrm{HR}=1.85 ; 95 \% \mathrm{CI}=1.16-2.95)$, and disease-specific survival $(\mathrm{HR}=1.33 ; 95 \% \mathrm{CI}=1.10-1.60)$ in gastric cancer. Conclusions. Our meta-analysis showed that CA 19-9 indicates clinicopathologic characteristics of gastric cancer and is associated with a poor prognosis.

\section{Introduction}

Gastric cancer remains the fourth most commonly diagnosed cancer and third leading cause of cancer deaths worldwide [1]. Gastric cancer is often diagnosed at an advanced stage and the survival rate is low [2]. The tumor-node-metastasis (TNM) classification is the most important prognostic factor in gastric cancer, but it is still difficult to obtain complete prognostic information [3]. Therefore, it is important to identify other markers, which should be simple, feasible, and less costly, for the assessment of clinicopathologic characteristics and prediction of prognosis.

As a type of tumor-associated antigen for gastrointestinal cancer, carbohydrate antigen (CA) 19-9 is a sialylated derivative of Lewis ${ }^{\mathrm{a}}$ blood group antigen [4] and is not applied to TNM staging of gastric cancer according to the American Joint Committee on Cancer (7th edition) [5]. Most research has focused on pancreatic cancer [6-8]. Therefore, the clinical value of CA 19-9 in gastric cancer remains controversial and has not been confirmed [9-11].

The aim of this meta-analysis was to evaluate the relationship between CA 19-9 and clinicopathologic characteristics and the prognostic value of CA 19-9 in gastric cancer.

\section{Materials and Methods}

2.1. Literature Search. A systematic literature search was conducted for relevant studies using PubMed and Embase databases. The search strategy included the following terms: "carbohydrate antigen 19-9," "carbohydrate antigen 199," "CA 19-9," “CA 199," "gastric cancer," and "stomach cancer." A manual search of the reference lists of the retrieved studies and reviews was also performed to identify potential studies.

2.2. Eligibility Criteria. Studies were included if the following inclusion criteria were met: (1) sample for measuring CA 
19-9 was obtained from serum of gastric cancer; (2) studies reported the clinicopathologic or/and prognostic values of CA 19-9; and (3) outcome measures could be extracted directly or calculated from published data indirectly. Only the most informative study was included if there were several duplicated studies based on the same patient population.

2.3. Data Extraction and Quality Assessment. Studies were reviewed and data were independently extracted by two reviewers (Yong-xi Song and Xuan-zhang Huang). The following data were extracted: first author; country and year of publication; sample size; patients characteristics; followup period; sampling time; cut-off value; and tumor clinicopathologic characteristics and prognostic value (overall survival (OS), disease-free survival (DFS), and disease-specific survival (DSS)). The quality of the included studies was assessed by Newcastle-Ottawa Scale (NOS) criteria [12]. Any disagreements on the data extraction and quality assessment were resolved by comprehensive discussion.

2.4. Statistical Analysis. Odds ratios (ORs) and 95\% confidence intervals (CIs) were used as a measure to evaluate the relationship between CA 19-9 and tumor clinicopathologic characteristics. For the relationship between CA 19-9 and prognosis, we used hazard ratios (HRs) and 95\% CIs as effect measures. HRs and 95\% CIs were extracted directly or calculated from available data using the methods designed by Tierney if the values were not reported directly [13]. For the studies that reported several results separately based on different subgroups, we pooled multiple effect values into an estimated value for further meta-analysis.

The Cochran $Q$ test and $I^{2}$ statistics were used to evaluate heterogeneity, and statistically significant heterogeneity was defined as $p<0.10$ and/or $I^{2}>50 \%$ [14]. We used a random effects model if significant heterogeneity existed; otherwise, a fixed effects model was used. The sources of heterogeneity were explored by metaregression and subgroup analyses. Metaregression was only performed when the number of studies was greater than ten, considering the accuracy of the results $[15,16]$. Publication bias was assessed using Begg's and Egger's tests, and trim-and-fill analysis was performed to assess the effect of publication bias [17-19].

All statistical analyses were conducted using STATA software (version 12.0; Stata Corporation, College Station, TX, USA), and a two-sided $p$ value $<0.05$ was considered statistically significant.

\section{Results}

3.1. Baseline Characteristics of Eligible Studies. A total of 1244 studies were initially identified, of which 943 were excluded after reviewing titles and abstracts. Then, 263 studies were excluded after full-text review. Finally, 38 studies were included (Figure 1) [9-11, 20-54].

The 38 eligible studies were published between 1995 and 2014 and included 11408 gastric cancer patients (mean sample size, 300; median sample size with corresponding range, 167 [23-1710]). Of the eligible studies, 30 assessed the value of

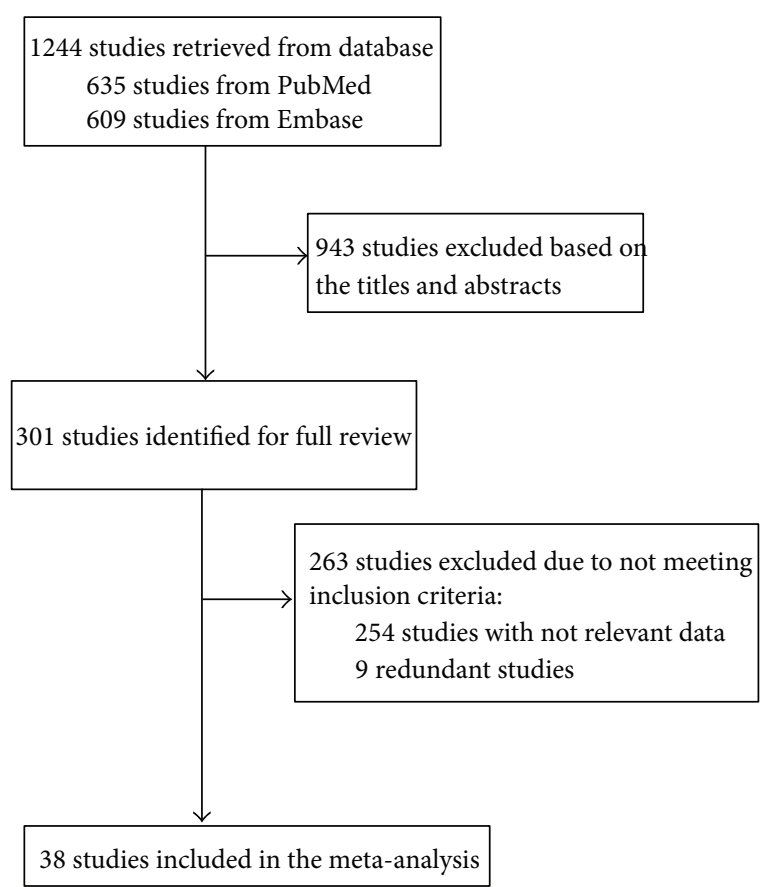

Figure 1: Flow diagram showing the literature search and selection process for the included studies.

preoperative CA $19-9[9,11,20,23,25-27,29-31,33,35-$ $47,49-54]$, two assessed the value of postoperative CA 19-9 $[28,34]$, two assessed the pre- and postoperative combined value of CA 19-9 [10,48], and four studies did not report sampling time [21, 22, 24, 32]. HRs for OS, DFS, and DSS were available in 29 [9-11, 20-24, 26-33, 35, 36, 38, 40, 44$47,49,50,52-54]$, seven $[9,11,29,34,36,43,48]$, and six studies [25, 37, 39, 41, 42, 51], respectively. The characteristics and quality of the included studies are shown in Table 1.

\subsection{Impact of CA 19-9 on Survival}

3.2.1. CA 19-9 and OS. Twenty-nine studies evaluated the prognostic effect of CA 19-9 on OS [9-11, 20-24, 26-33, $35,36,38,40,44-47,49,50,52-54]$. Our results indicated that the high CA 19-9 group had a significantly shorter OS $\left(\mathrm{HR}=1.83\right.$; 95\% CI $=1.56-2.15 ; I^{2}=75.8 \%$; Figure 2$)$. The result of subgroup analysis for preoperative CA $19-9$ was similar $\left(\mathrm{HR}=1.87 ; 95 \% \mathrm{CI}=1.52-2.30 ; I^{2}=76.3 \%\right)$. As shown by the subgroup analyses stratified by cut-off value ( $37 \mathrm{U} / \mathrm{mL}$ and other than $37 \mathrm{U} / \mathrm{mL}$ ) and study quality (NOS $\geq 6$ and NOS $<6$ ), the prognostic effect of CA 19-9 on OS was confirmed (Table 2).

3.2.2. CA 19-9 and DFS. Seven studies evaluated the prognostic effect of CA 19-9 on DFS [9, 11, 29, 34, 36, 43, 48]. Our results indicated that a significantly poor DFS existed in the high CA 19-9 group ( $\mathrm{HR}=1.85 ; 95 \% \mathrm{CI}=1.16-2.95 ; I^{2}=$ $60.2 \%$; Figure 3). The results for preoperative CA 19-9 levels were marginally significant $(\mathrm{HR}=1.96$; 95\% $\mathrm{CI}=1.00-3.85$; $I^{2}=72.9 \%$ ). Similarly, subgroup analyses for cut-off value 


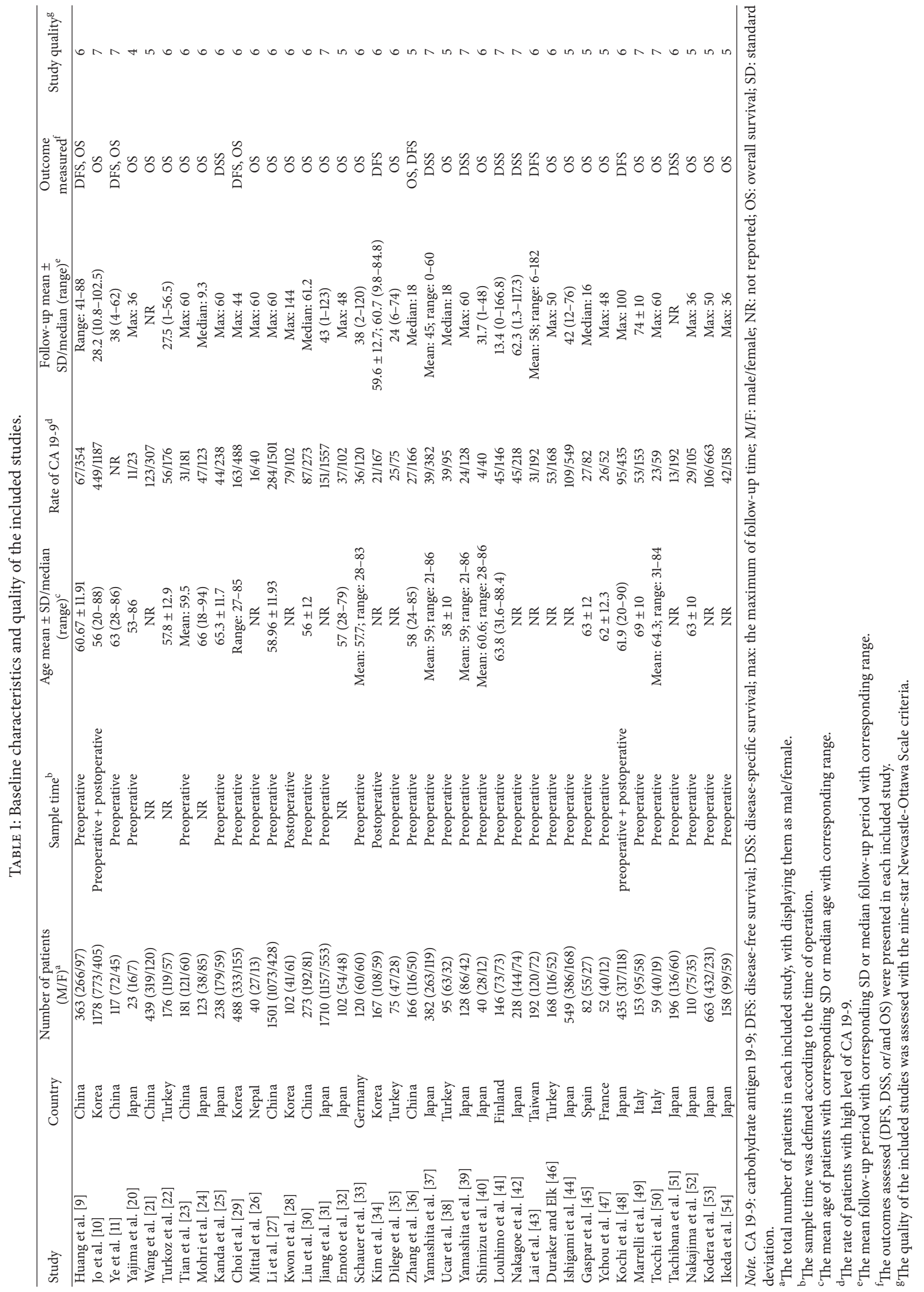




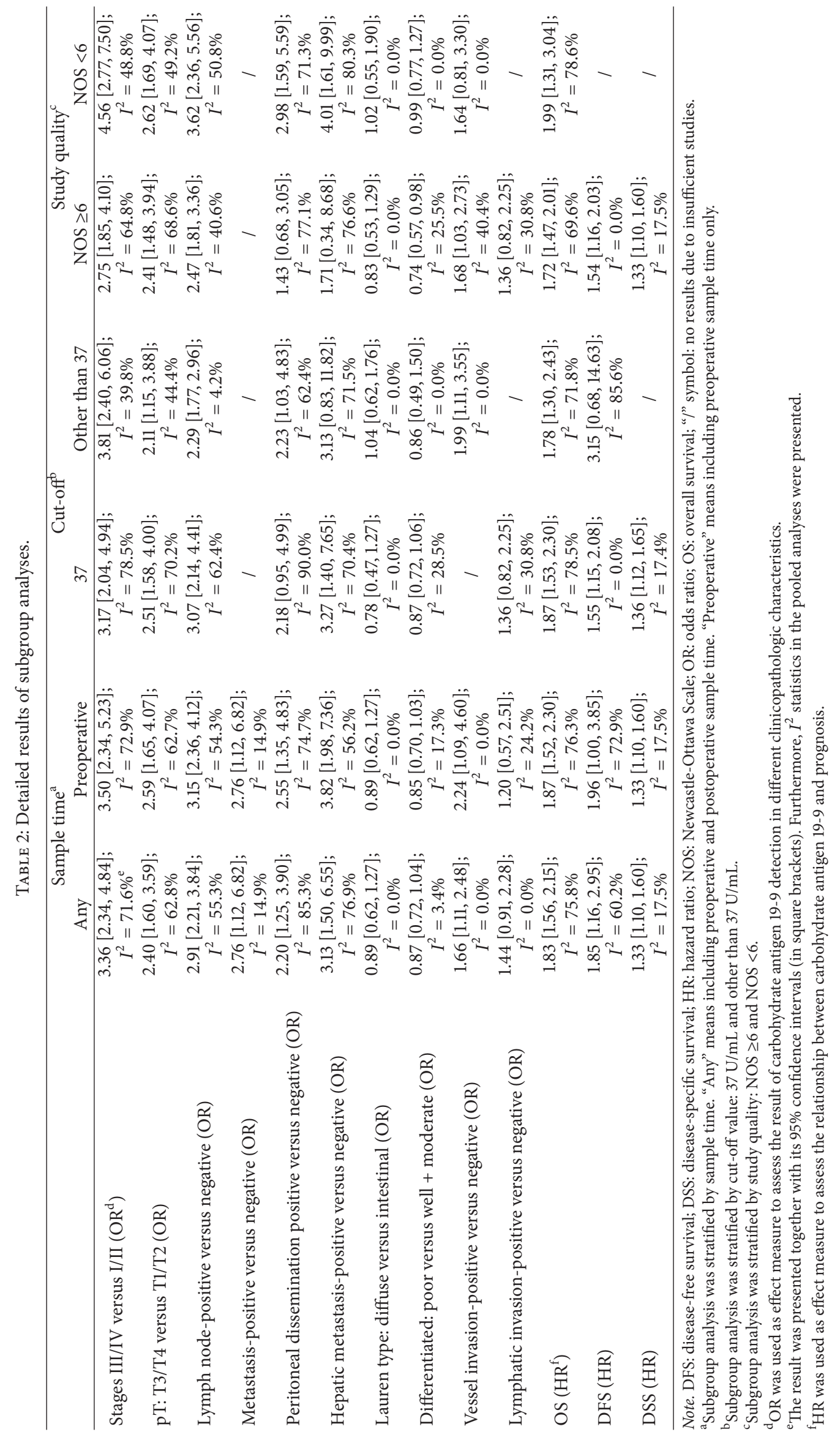




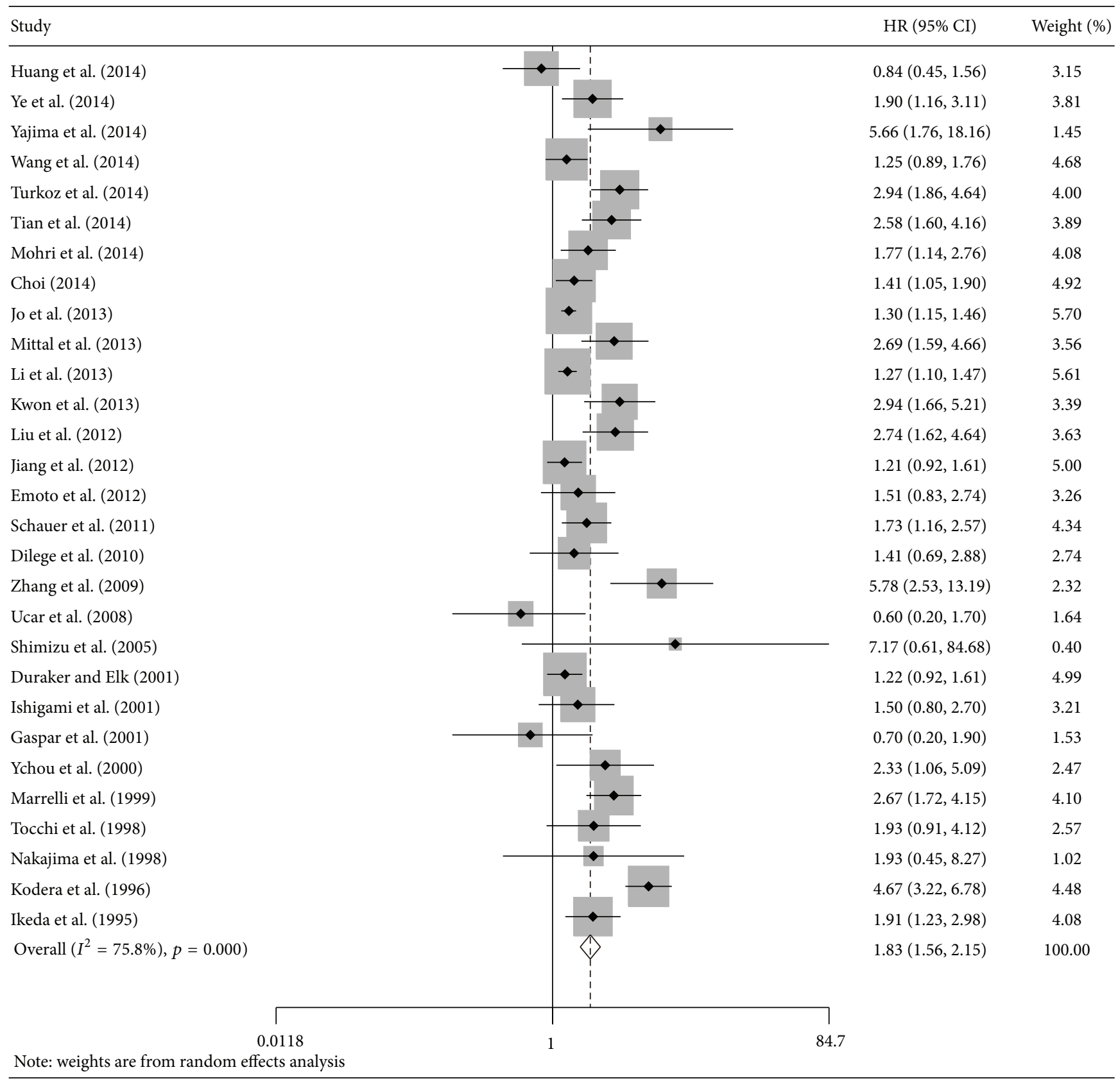

FIGURE 2: The estimated hazard ratio (HR) was summarized for the association between carbohydrate antigen 19-9 and overall survival. The left-hand column lists the first author of each study; the middle column graphically displays the effect measure for each study incorporating confidence intervals (the black solid point represents effect measure and horizontal line represents $95 \%$ confidence intervals), the area of gray square is proportional to the study's weight in the meta-analysis, and the pooled measure of effect is plotted as a diamond; the right-hand column numerically displays the effect measure, $95 \%$ confidence intervals, and weight of each study.

and NOS score also showed that CA 19-9 was a significant poor prognostic factor for DFS (Table 2).

3.2.3. CA 19-9 and DSS. Six studies evaluated the prognostic effect of CA 19-9 on DSS [25, 37, 39, 41, 42, 51]. Our results indicated that the high CA 19-9 group was associated with a significantly poor DSS (HR $=1.33$; $95 \% \mathrm{CI}=1.10-1.60$; $I^{2}=17.5 \%$; Figure 4). Including the studies using a cut-off value of $37 \mathrm{U} / \mathrm{mL}$, we observed a similar result (Table 2).

\subsection{Relationship between CA 19-9 and Clinicopathologic Characteristics}

3.3.1. CA 19-9 and TNM Stage. There were 17 studies that provided data on TNM stage and CA $19-9[9,20,23,29,35$, $36,38,41-43,45,46,48,50,52-54]$. Our results indicated a significantly higher incidence of high CA 19-9 levels in stages III/IV group relative to stages I/II group $(\mathrm{OR}=3.36$; 


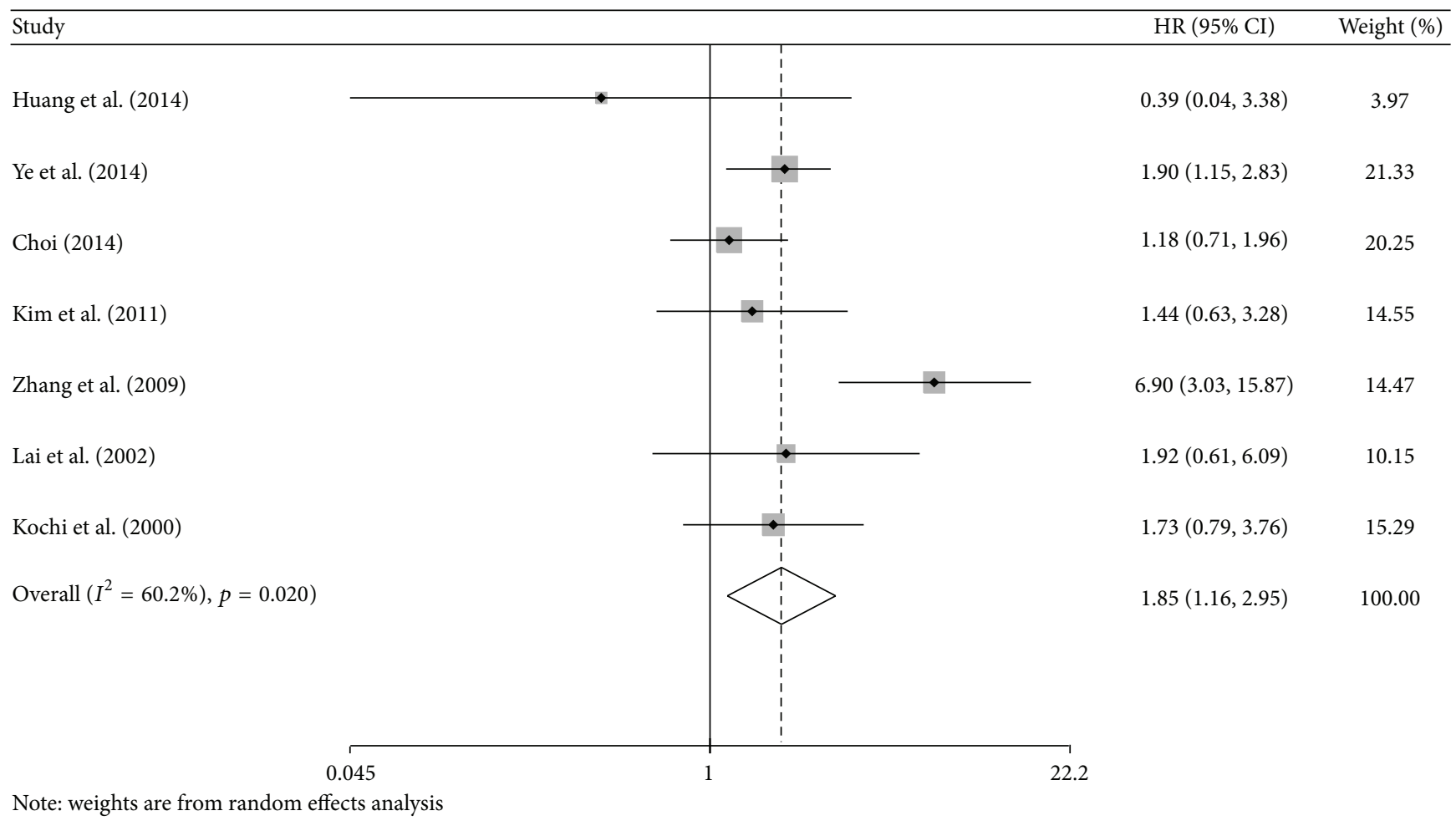

FIGURE 3: The estimated hazard ratio (HR) was summarized for the association between carbohydrate antigen 19-9 and disease-free survival. The left-hand column lists the first author of each study; the middle column graphically displays the effect measure for each study incorporating confidence intervals (the black solid point represents effect measure and horizontal line represents $95 \%$ confidence intervals), the area of gray square is proportional to the study's weight in the meta-analysis, and the pooled measure of effect is plotted as a diamond; the right-hand column numerically displays the effect measure, $95 \%$ confidence intervals, and weight of each study.

95\% CI $=2.34-4.84 ; I^{2}=71.6 \%$; Figure 5). Moreover, a similar tendency was obtained in the subgroup analysis based on preoperative time, cut-off value, and study quality (Table 2).

There were significant differences in the incidence of high CA 19-9 levels between the pT3/T4 and pT1/T2 groups $\left(\mathrm{OR}=2.40 ; 95 \% \mathrm{CI}=1.60-3.59 ; I^{2}=62.8 \%\right)$, the lymph node-positive and lymph node-negative groups (OR = 2.91; $95 \%$ CI $=2.21-3.84 ; I^{2}=55.3 \%$ ), and the metastasispositive and metastasis-negative groups (OR $=2.76 ; 95 \%$ $\mathrm{CI}=1.12-6.82 ; I^{2}=14.9 \%$; Table 2 ).

A higher incidence of high CA 19-9 levels was observed in the peritoneal $\left(\mathrm{OR}=2.20 ; 95 \% \mathrm{CI}=1.25-3.90 ; I^{2}=\right.$ $85.3 \%$ ) and hepatic metastasis-positive groups (OR = 3.13; $\left.95 \% \mathrm{CI}=1.50-6.55 ; I^{2}=76.9 \%\right)$.

3.3.2. CA 19-9 and Vessel and Lymphatic Invasion. Four [21, $35,48,52]$ and three studies $[48,50,52]$ assessed the relationship between CA 19-9 and vessel invasion and between CA 19-9 and lymphatic invasion, respectively. Our results showed a significantly higher incidence of high CA 19-9 levels in the vessel invasion-positive group compared with the vessel invasion-negative group, and there was no significant relationship between the CA 19-9 level and lymphatic invasion (Table 2).
3.3.3. CA 19-9 and Histologic Type. Seventeen studies assessed the relationship between the CA 19-9 level and histologic type [9, 20, 21, 23, 28, 29, 35, 38, 42-46, 49, 52-54]. The results indicated that there was no significant relationship between CA 19-9 and tumor differentiation (Lauren diffuse type versus intestinal type: $\mathrm{OR}=0.89 ; 95 \% \mathrm{CI}=0.62-1.27$; $I^{2}=0.0 \%$; poor differentiation versus well/moderate differentiation: $\left.\mathrm{OR}=0.87 ; 95 \% \mathrm{CI}=0.72-1.04 ; I^{2}=3.4 \%\right)$. As shown by the subgroup analyses stratified by sampling time, cut-off value, and study quality, similar tendencies were observed.

3.4. Assessment of Publication Bias and Heterogeneity. The results of Begg's and Egger's tests showed no significant publication bias, except in the HRs for OS. The trim-andfill analyses indicated that publication bias could not impact on the results for OS. Metaregression analyses showed that publication year may contribute to heterogeneity in the analyses of lymph node and hepatic metastases (lymph node: coefficient $=-0.041$, standard error $=0.019$, and $p=0.046$; hepatic metastasis: coefficient $=-0.113$, standard error $=$ 0.021 , and $p=0.001$ ).

\section{Discussion}

Gastric cancer is a global health problem with a low survival rate. Tumor-associated markers are urgently needed for the 


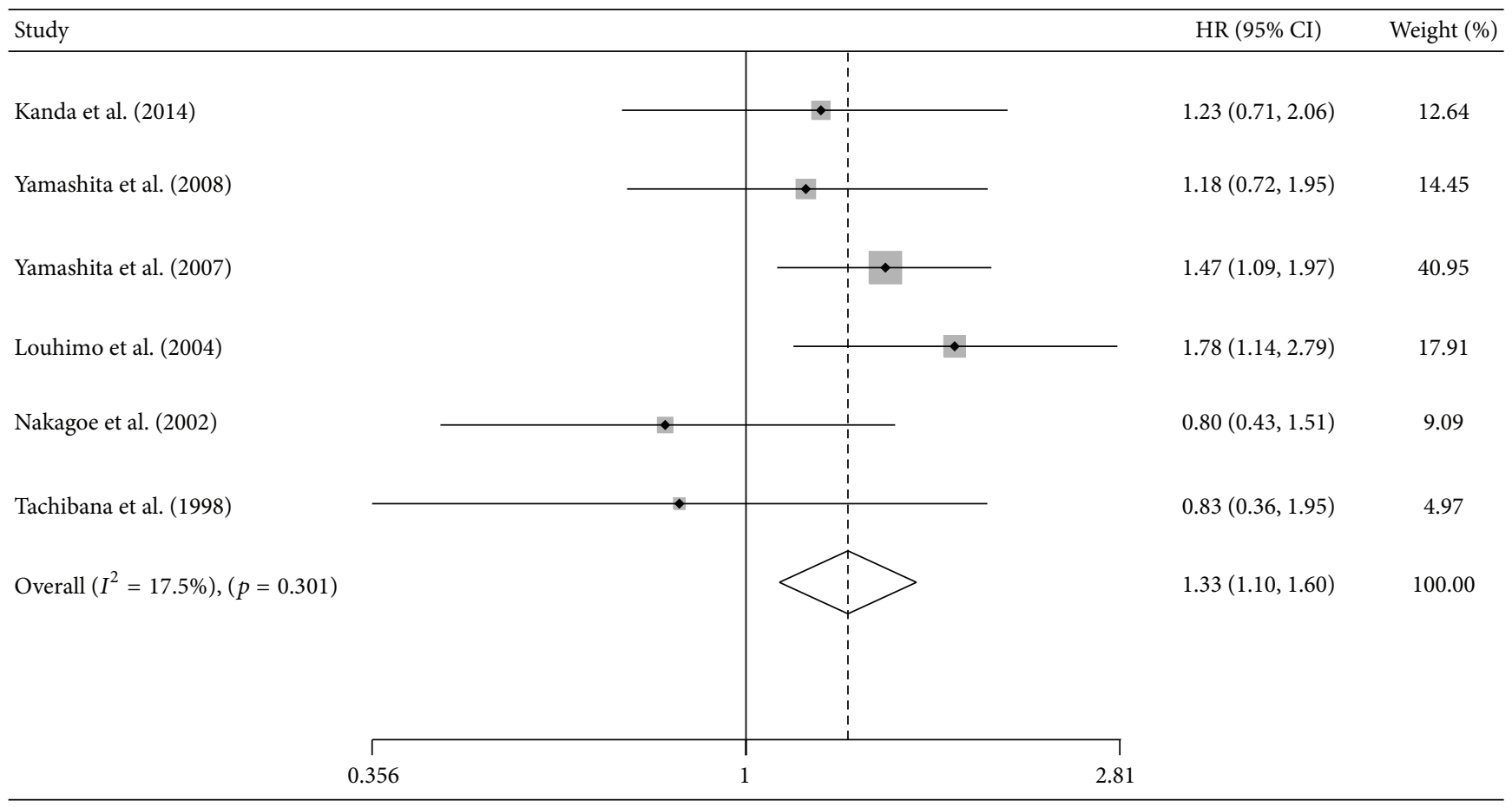

FIGURE 4: The estimated hazard ratio (HR) was summarized for the association between carbohydrate antigen 19-9 and disease-specific survival. The left-hand column lists the first author of each study; the middle column graphically displays the effect measure for each study incorporating confidence intervals (the black solid point represents effect measure and horizontal line represents $95 \%$ confidence intervals), the area of gray square is proportional to the study's weight in the meta-analysis, and the pooled measure of effect is plotted as a diamond; the right-hand column numerically displays the effect measure, 95\% confidence intervals, and weight of each study.

diagnosis of cancer and assessment of prognosis. Nevertheless, gastric cancer-specific markers have not yet been established, and the unsatisfactory specificity and sensitivity limit clinical utility. Although many studies have been conducted to evaluate the clinical value of CA 19-9 in gastric cancer, there is still no general agreement.

Our meta-analysis provides important and valuable evidence for the individualized treatment for gastric cancer. Our results indicate that CA 19-9 is associated with clinicopathologic characteristics, including tumor stage, $\mathrm{pT}$ category, lymph node metastasis, distant metastasis, and vessel invasion, and is feasible for gastric tumor staging. Moreover, our results provide evidence that CA 19-9 can be used to predict the prognosis of gastric cancer. Similar results were obtained in the subgroup analyses stratified by sample time, cut-off value, and study quality.

Recently, several studies have reported that CA 19-9 is associated with gastric cancer metastasis and prognosis [24, 49]. Our results also obtained similar evidence. Metastatic patients had a 2.76-fold elevated level of CA 19-9 compared to patients without metastasis. Moreover, the CA 19-9 level was more frequently elevated in patients with peritoneal and hepatic metastases (2.20- and 3.13-fold, resp.). Our results also indicated that an elevated level of CA 19-9 is associated with poor prognosis in gastric cancer, including poor OS, DFS, and DSS. Marrelli et al. demonstrated that stage I patients with elevated CA 19-9 levels had a similar prognosis to stage II with normal levels, and the prognosis of stage
II with elevated levels was similar to stage III with normal levels [49]. Thus, the CA 19-9 level had a very important and valuable prognostic value in patients with gastric cancer. Nevertheless, the intrinsic mechanisms by which the elevated level of CA 19-9 can result in poor prognosis are unclear. The most plausible explanation may be that CA 19-9, as a ligand of E-selectin and an intercellular adhesion molecule, plays a crucial role in intercellular adhesion of tumor cells to vascular endothelial cells and then contributes to tumor invasion and metastasis $[55,56]$. Indeed, experimental studies have demonstrated that cells expressing CA 19-9 may have a greater capacity of invasion and metastasis [57-59]. In addition, Tabuchi et al. suggested that CA 19-9 may be drained by the thoracic duct of the lymphatic system via node metastasis or invasive lymphatics [60]; thus the elevated level of CA 19-9 may prompt the presence of micrometastases, which can lead to subsequent relapse/metastasis. Further studies are needed to investigate the potential mechanisms underlying the association between CA 19-9 and tumor metastasis and prognosis.

In our meta-analysis, the elevated level rate (sensitivity) of CA $19-9$ ranges from $6.8 \%$ to $51.7 \%$ (median: $30.0 \%$ ) and the CA 19-9 level was most frequently elevated in advanced stage tumor. Kochi et al. reported that the elevated level of CA $19-9$ increased gradually with tumor stages $25.3 \%$ in stage I, $10.5 \%$ in stage II, $12.6 \%$ in stage III, and $51.6 \%$ in stage IV) [48]. Similarly, Duraker and Elk reported that the rate of advanced tumors (stages III and IV) was significantly higher 


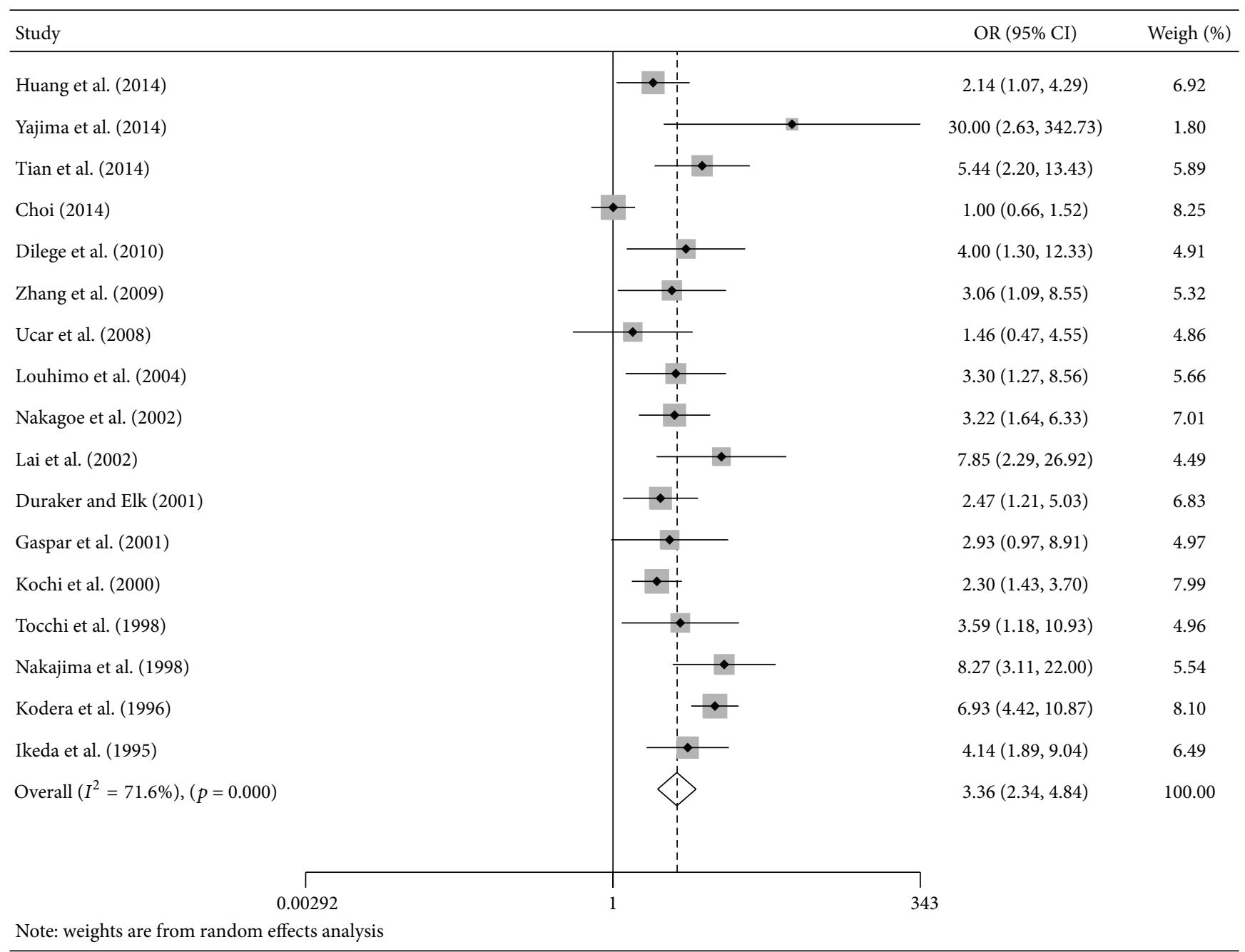

FIgURE 5: The estimated odds ratio (OR) was summarized for the association between carbohydrate antigen 19-9 and tumor stage. The lefthand column lists the first author of each study; the middle column graphically displays the effect measure for each study incorporating confidence intervals (the black solid point represents effect measure and horizontal line represents $95 \%$ confidence intervals), the area of gray square is proportional to the study's weight in the meta-analysis, and the pooled measure of effect is plotted as a diamond; the right-hand column numerically displays the effect measure, $95 \%$ confidence intervals, and weight of each study.

in patients with elevated CA 19-9 levels than in patients with normal levels [46]. The included patients in the Tachibana et al., Jiang et al., Shimizu et al., Yamashita et al., and Kim et al. studies were mostly early stage $[31,34,37,40,51]$, and this may be the main reason why the elevated CA 19-9 level was low $(6.8 \%-12.6 \%)$. In contrast, other studies included advanced stage or metastatic patients, and the elevated CA 19-9 level was high $(36.3 \%-54.1 \%)$ [10, 21, 22, 24, 28, 32, 47]. Thus, the CA 19-9 levels may reflect tumor burden and indicate tumor stage preoperatively. Future studies are needed to explore whether the clinical values of CA 19-9 levels differ in patients of different tumor stages.

However, insufficient sensitivity of CA 19-9 may limit its clinical utility; a number of studies have been conducted to resolve the problem via increasing sensitivity. Ikeda et al. reported that combination of CA 19-9 and other tumor markers could provide more useful diagnostic information for patients with gastric cancer than CA 19-9 alone [54].
Similarly, Tian et al. reported the complementary role of CA 19-9 and carcinoembryonic antigen (CEA), and the sensitivity increased with combined use [23]. Future studies are needed to explore which kinds of tumor markers can be combined with CA 19-9 to increase diagnostic accuracy obviously.

Compared with the diagnostic value, the sensitivity for predicting recurrence was relatively higher. Marrelli et al. reported that the sensitivity for recurrence of CA 19-9 was $56 \%$, with a specificity of $74 \%$ [61]. Kim et al. reported that the sensitivity for recurrence was $68.2 \%$ in advanced gastric cancer, with a specificity of $80.0 \%$ [34]. Moreover, the combination of CA 19-9 and other tumor markers provided more useful prognostic information. Marrelli et al. reported that the sensitivity increased to $87 \%$ when CA $19-9$ was combined with CEA and CA 72-4 [61]. Huang et al. obtained similar results on the prediction of recurrence [9]. Thus, CA 19-9, a low-cost and convenient test, may be useful for 
predicting prognosis and of use as an important indicator for high risk of recurrence.

There is no agreement on the optimal cut-off value for the prognostic value of CA 19-9. Most studies have used a cut-off value of $37 \mathrm{U} / \mathrm{mL}$, but this cut-off value is often used for the diagnosis of gastric cancer and it is unknown whether this cut-off value is appropriate as a prognostic value. Yajima et al. determined the cut-off value of CA 19-9 as a predictor of prognosis via receiver operating characteristic analysis, and the cut-off value was $\geq 77 \mathrm{U} / \mathrm{mL}$, which was approximately twice the upper limit of the normal range $(37 \mathrm{U} / \mathrm{mL})$ [20]. High-quality, well-designed multicenter studies are required to establish an optimal cut-off value for CA 19-9 and determine whether we should use different cut-off values for clinicopathologic and prognostic values.

The strength of the present meta-analysis lies in the inclusion of all relevant studies. Not only did we evaluate the clinicopathologic value of CA 19-9 in patients with gastric cancer, we also assessed the prognostic value of CA 19-9. Moreover, our results and conclusions were confirmed by indepth subgroup analyses. Thus, our results may be valuable for diagnosis, prediction of prognosis, and individual treatment in patient with gastric cancer.

There were several limitations in the present study. First, we could not obtain detailed individual information. Thus, we could not control other potential biases. Second, there was considerable heterogeneity and heterogeneity still could not be eliminated. Third, our study did not obtain a conclusive result regarding the optimal cut-off value and could not evaluate whether chemotherapy would impact the prognostic value of CA 19-9 due to a limited number of included studies. Therefore, future studies are urgently needed to assess the cut-off value of CA 19-9 and effects of chemotherapy on CA 19-9.

\section{Conclusions}

Our meta-analysis showed that CA 19-9 may indicate clinicopathologic characteristics of gastric cancer and is associated with poor prognosis. Further high-quality and large-scale studies are required to determine whether CA 19-9 can be used for the individualized treatment for patients with gastric cancer.

\section{Ethical Approval}

The study did not violate the local regulations and was approved by the Research Ethics Committee of China Medical University, China.

\section{Conflict of Interests}

The authors declare that there is no conflict of interests regarding the publication of this paper.

\section{Acknowledgments}

The authors thank the Department of Surgical Oncology of First Hospital of China Medical University and the College of China Medical University for technical assistance. This work was supported by Natural Science Foundation of Liaoning Province (no. 2014029201), Program of Education Department of Liaoning Province (L2014307), and Ministry of Education (IRT13101).

\section{References}

[1] L. A. Torre, F. Bray, R. L. Siegel, J. Ferlay, J. Lortet-Tieulent, and A. Jemal, "Global cancer statistics, 2012," CA Cancer Journal for Clinicians, vol. 65, no. 2, pp. 87-108, 2015.

[2] P. Karimi, F. Islami, S. Anandasabapathy, N. D. Freedman, and F. Kamangar, "Gastric cancer: descriptive epidemiology, risk factors, screening, and prevention," Cancer Epidemiology Biomarkers \& Prevention, vol. 23, no. 5, pp. 700-713, 2014.

[3] S. B. Edge and C. C. Compton, "The american joint committee on cancer: the 7th edition of the AJCC cancer staging manual and the future of TNM," Annals of Surgical Oncology, vol. 17, no. 6, pp. 1471-1474, 2010.

[4] J. L. Magnani, B. Nilsson, M. Brockhaus et al., "A monoclonal antibody-defined antigen associated with gastrointestinal cancer is a ganglioside containing sialylated lacto- $\mathrm{N}$-fucopentaose II," The Journal of Biological Chemistry, vol. 257, no. 23, pp. 14365-14369, 1982.

[5] K. Washington, "7th edition of the AJCC cancer staging manual: stomach," Annals of Surgical Oncology, vol. 17, no. 12, pp. 30773079, 2010.

[6] T. M. Bauer, B. F. El-Rayes, X. Li et al., "Carbohydrate antigen 19-9 is a prognostic and predictive biomarker in patients with advanced pancreatic cancer who receive gemcitabinecontaining chemotherapy: a pooled analysis of 6 prospective trials," Cancer, vol. 119, no. 2, pp. 285-292, 2013.

[7] J. Kim, Y. S. Lee, I. K. Hwang et al., "Postoperative carcinoembryonic antigen as a complementary tumor marker of carbohydrate antigen 19-9 in pancreatic ductal adenocarcinoma," Journal of Korean Medical Science, vol. 30, no. 3, pp. 259-263, 2015.

[8] X. G. Ni, X. F. Bai, Y. L. Mao et al., "The clinical value of serum CEA, CA19-9, and CA242 in the diagnosis and prognosis of pancreatic cancer," European Journal of Surgical Oncology, vol. 31, no. 2, pp. 164-169, 2005.

[9] Z. B. Huang, X. Zhou, J. Xu et al., "Prognostic value of preoperative serum tumor markers in gastric cancer," World Journal of Clinical Oncology, vol. 5, no. 2, pp. 170-176, 2014.

[10] J.-C. Jo, M.-H. Ryu, D.-H. Koo et al., "Serum CA 19-9 as a prognostic factor in patients with metastatic gastric cancer," Asia-Pacific Journal of Clinical Oncology, vol. 9, no. 4, pp. 324330, 2013.

[11] X.-T. Ye, A.-J. Guo, P.-F. Yin, X.-D. Cao, and J.-C. Chang, "Overexpression of NUAK1 is associated with disease-free survival and overall survival in patients with gastric cancer," Medical Oncology, vol. 31, no. 7, article 61, 2014.

[12] A. Stang, "Critical evaluation of the Newcastle-Ottawa scale for the assessment of the quality of nonrandomized studies in metaanalyses," European Journal of Epidemiology, vol. 25, no. 9, pp. 603-605, 2010

[13] J. F. Tierney, L. A. Stewart, D. Ghersi, S. Burdett, and M. R. Sydes, "Practical methods for incorporating summary time-toevent data into meta-analysis," Trials, vol. 8, article 16, 2007.

[14] J. P. T. Higgins, S. G. Thompson, J. J. Deeks, and D. G. Altman, "Measuring inconsistency in meta-analyses," British Medical Journal, vol. 327, no. 7414, pp. 557-560, 2003. 
[15] C. H. Schmid, P. C. Stark, J. A. Berlin, P. Landais, and J. Lau, "Meta-regression detected associations between heterogeneous treatment effects and study-level, but not patient-level, factors," Journal of Clinical Epidemiology, vol. 57, no. 7, pp. 683-697, 2004.

[16] J. Higgins, S. Thompson, J. Deeks, and D. Altman, "Statistical heterogeneity in systematic reviews of clinical trials: a critical appraisal of guidelines and practice," Journal of Health Services Research \& Policy, vol. 7, no. 1, pp. 51-61, 2002.

[17] M. Egger, G. Davey Smith, M. Schneider, and C. Minder, "Bias in meta-analysis detected by a simple, graphical test," British Medical Journal, vol. 315, no. 7109, pp. 629-634, 1997.

[18] C. B. Begg and M. Mazumdar, "Operating characteristics of a rank correlation test for publication bias," Biometrics, vol. 50, no. 4, pp. 1088-1101, 1994.

[19] S. Duval and R. Tweedie, "Trim and fill: a simple funnel-plotbased method of testing and adjusting for publication bias in meta-analysis," Biometrics, vol. 56, no. 2, pp. 455-463, 2000.

[20] H. Yajima, N. Omura, K. Matai, N. Mitsumori, K. Yoshida, and K. Yanaga, "Clinicopathological features of CA19-9-producing gastric cancer," Hepato-Gastroenterology, vol. 61, no. 129, pp. 221-225, 2014.

[21] Q. Wang, Y. Yang, Y.-P. Zhang et al., "Prognostic value of carbohydrate tumor markers and inflammation-based markers in metastatic or recurrent gastric cancer," Medical Oncology, vol. 31, no. 12, article 289, 2014.

[22] F. P. Turkoz, M. Solak, S. Kilickap et al., "Bone metastasis from gastric cancer: the incidence, clinicopathological features, and influence on survival," Journal of Gastric Cancer, vol. 14, no. 3, pp. 164-172, 2014.

[23] S.-B. Tian, J.-C. Yu, W.-M. Kang et al., "Combined detection of CEA, CA 19-9, CA 242 and CA 50 in the diagnosis and prognosis of resectable gastric cancer," Asian Pacific Journal of Cancer Prevention, vol. 15, no. 15, pp. 6295-6300, 2014.

[24] Y. Mohri, K. Tanaka, M. Ohi et al., "Identification of prognostic factors and surgical indications for metastatic gastric cancer," BMC Cancer, vol. 14, article 409, 2014.

[25] M. Kanda, S. Nomoto, H. Oya et al., "Decreased expression of prenyl diphosphate synthase subunit 2 correlates with reduced survival of patients with gastric cancer," Journal of Experimental \& Clinical Cancer Research, vol. 33, no. 1, p. 88, 2014.

[26] A. Mittal, S. P. Gupta, D. K. Jha, B. Sathian, and B. Poudel, "Impact of various tumor markers in prognosis of gastric cancer. A hospital based study from tertiary care hospital of Kathmandu Valley," Asian Pacific Journal of Cancer Prevention, vol. 14, no. 3, pp. 1965-1967, 2013.

[27] F. Li, S. Li, L. Wei, X. Liang, H. Zhang, and J. Liu, "The correlation between pre-operative serum tumor markers and lymph node metastasis in gastric cancer patients undergoing curative treatment," Biomarkers, vol. 18, no. 7, pp. 632-637, 2013.

[28] O. K. Kwon, W. Yu, and H. Chung, "Prognostic value of postoperative CA19-9 normalization in patients with advanced gastric cancer," Hepato-Gastroenterology, vol. 60, no. 121, pp. 240-243, 2013.

[29] A. R. Choi, J. C. Park, J.-H. Kim et al., "High level of preoperative carbohydrate antigen 19-9 is a poor survival predictor in gastric cancer," World Journal of Gastroenterology, vol. 19, no. 32, pp. 5302-5308, 2013.

[30] X. Liu, H. Cai, and Y. Wang, "Prognostic significance of tumor markers in T4a gastric cancer," World Journal of Surgical Oncology, vol. 10, article 68, 2012.
[31] X. Jiang, N. Hiki, S. Nunobe et al., "Prognostic importance of the inflammation-based Glasgow prognostic score in patients with gastric cancer," British Journal of Cancer, vol. 107, no. 2, pp. 275-279, 2012.

[32] S. Emoto, H. Ishigami, H. Yamashita, H. Yamaguchi, S. Kaisaki, and J. Kitayama, "Clinical significance of CA125 and CA72-4 in gastric cancer with peritoneal dissemination," Gastric Cancer, vol. 15, no. 2, pp. 154-161, 2012.

[33] M. Schauer, M. Peiper, J. Theisen, and W. Knoefel, "Prognostic factors in patients with diffuse type gastric cancer (linitis plastica) after operative treatment," European Journal of Medical Research, vol. 16, no. 1, pp. 29-33, 2011.

[34] D. H. Kim, S. J. Oh, C. A. Oh et al., "The relationships between perioperative CEA, CA 19-9, and CA 72-4 and recurrence in gastric cancer patients after curative radical gastrectomy," Journal of Surgical Oncology, vol. 104, no. 6, pp. 585-591, 2011.

[35] E. Dilege, M. Mihmanli, U. Demir et al., "Prognostic value of preoperative CEA and CA 19-9 levels in resectable gastric cancer," Hepato-Gastroenterology, vol. 57, no. 99-100, pp. 674$677,2010$.

[36] Y.-H. Zhang, Y. Li, C. Chen, and C.-W. Peng, "Carcinoembryonic antigen level is related to tumor invasion into the serosa of the stomach: study on 166 cases and suggestion for new therapy," Hepato-Gastroenterology, vol. 56, no. 96, pp. 1750-1754, 2009.

[37] K. Yamashita, A. Ooki, S. Sakuramoto et al., "Lymph node metastasis density (ND)-factor association with malignant degree and ND40 as 'non-curative factor' in gastric cancer," Anticancer Research, vol. 28, no. 1, pp. 435-441, 2008.

[38] E. Ucar, E. Semerci, H. Ustun, T. Yetim, C. Huzmeli, and M. Gullu, "Prognostic value of preoperative CEA, CA 19-9, CA 724, and AFP levels in gastric cancer," Advances in Therapy, vol. 25, no. 10, pp. 1075-1084, 2008.

[39] K. Yamashita, S. Sakuramoto, S. Kikuchi, N. Katada, N. Kobayashi, and M. Watanabe, "Surgical resection of stage IV gastric cancer and prognosis," Anticancer Research, vol. 27, no. 6 C, pp. 4381-4386, 2007.

[40] K. Shimizu, Y. Ueda, and H. Yamagishi, "Titration of serum p53 antibodies in patients with gastric cancer: a single-institute study of 40 patients," Gastric Cancer, vol. 8, no. 4, pp. 214-219, 2005.

[41] J. Louhimo, A. Kokkola, H. Alfthan, U.-H. Stenman, and C. Haglund, "Preoperative hCG $\beta$ and CA 72-4 are prognostic factors in gastric cancer," International Journal of Cancer, vol. 111, no. 6, pp. 929-933, 2004.

[42] T. Nakagoe, T. Sawai, T. Tsuji et al., "Difference in prognostic value between sialyl Lewisa and sialyl Lewisx antigen levels in the preoperative serum of gastric cancer patients," Journal of Clinical Gastroenterology, vol. 34, no. 4, pp. 408-415, 2002.

[43] I.-R. Lai, W.-J. Lee, M.-T. Huang, and H.-H. Lin, “Comparison of serum CA72-4, CEA, TPA, CA19-9 and CA125 levels in gastric cancer patients and correlation with recurrence," HepatoGastroenterology, vol. 49, no. 46, pp. 1157-1160, 2002.

[44] S. Ishigami, S. Natsugoe, S. Hokita et al., "Clinical importance of preoperative carcinoembryonic antigen and carbohydrate antigen 19-9 levels in gastric cancer," Journal of Clinical Gastroenterology, vol. 32, no. 1, pp. 41-44, 2001.

[45] M. J. Gaspar, I. Arribas, M. C. Coca, and M. Díez-Alonso, "Prognostic value of carcinoembryonic antigen, CA 19-9 and CA 72-4 in gastric carcinoma," Tumor Biology, vol. 22, no. 5, pp. 318-322, 2001. 
[46] N. Duraker and A. N. Elk, "The prognostic significance of preoperative serum CA 19-9 in patients with resectable gastric carcinoma: comparison with CEA," Journal of Surgical Oncology, vol. 76, no. 4, pp. 266-271, 2001.

[47] M. Ychou, J. Duffour, A. Kramar, S. Gourgou, and J. Grenier, "Clinical significance and prognostic value of CA72-4 compared with CEA and CA19-9 in patients with gastric cancer," Disease Markers, vol. 16, no. 3-4, pp. 105-110, 2000.

[48] M. Kochi, M. Fujii, N. Kanamori et al., "Evaluation of serum CEA and CA19-9 levels as prognostic factors in patients with gastric cancer," Gastric Cancer, vol. 3, no. 4, pp. 177-186, 2000.

[49] D. Marrelli, F. Roviello, A. De Stefano et al., "Prognostic significance of CEA, CA 19-9 and CA 72-4 preoperative serum levels in gastric carcinoma," Oncology, vol. 57, no. 1, pp. 55-62, 1999.

[50] A. Tocchi, G. Costa, L. Lepre et al., "The role of serum and gastric juice levels of carcinoembryonic antigen, CA19.9 and CA72.4 in patients with gastric cancer," Journal of Cancer Research and Clinical Oncology, vol. 124, no. 8, pp. 450-455, 1998.

[51] M. Tachibana, Y. Takemoto, Y. Nakashima et al., "Serum carcinoembryonic antigen as a prognostic factor in resectable gastric cancer," Journal of the American College of Surgeons, vol. 187, no. 1, pp. 64-68, 1998.

[52] K. Nakajima, T. Ochiai, T. Suzuki et al., "Impact of preoperative serum carcinoembryonic antigen, CA 19-9 and alpha fetoprotein levels in gastric cancer patients," Tumor Biology, vol. 19, no. 6, pp. 464-469, 1998.

[53] Y. Kodera, Y. Yamamura, A. Torii et al., "The prognostic value of preoperative serum levels of CEA and CA19-9 in patients with gastric cancer," The American Journal of Gastroenterology, vol. 91, no. 1, pp. 49-53, 1996.

[54] Y. Ikeda, H. Oomori, N. Koyanagi et al., "Prognostic value of combination assays for CEA and CA 19-9 in gastric cancer," Oncology, vol. 52, no. 6, pp. 483-486, 1995.

[55] J. B. Lowe, L. M. Stoolman, R. P. Nair, R. D. Larsen, T. L. Berhend, and R. M. Marks, "ELAM-1-dependent cell adhesion to vascular endothelium determined by a transfected human fucosyltransferase cDNA," Cell, vol. 63, no. 3, pp. 475-484, 1990.

[56] E. L. Berg, M. K. Robinson, O. Mansson, E. C. Butcher, and J. L. Magnani, "A carbohydrate domain common to both sialyl $\mathrm{Le}^{\mathrm{a}}$ and sialyl $\mathrm{Le}^{\mathrm{X}}$ is recognized by the endothelial cell leukocyte adhesion molecule ELAM-1," The Journal of Biological Chemistry, vol. 266, no. 23, pp. 14869-14872, 1991.

[57] G. Yogeeswaran and P. L. Salk, "Metastatic potential is positively correlated with cell surface sialylation of cultured murine tumor cell lines," Science, vol. 212, no. 4502, pp. 1514-1516, 1981.

[58] G. Yogeeswaran, B. S. Stein, and H. Sebastian, "Altered cell surface organization of gangliosides and sialylglycoproteins of mouse metastatic melanoma variant lines selected in vivo for enhanced lung implantation," Cancer Research, vol. 38, no. 5, pp. 1336-1344, 1978.

[59] I. Kijima-Suda, Y. Miyamoto, S. Toyoshima, M. Itoh, and T. Osawa, "Inhibition of experimental pulmonary metastasis of mouse colon adenocarcinoma 26 sublines by a sialic acid: nucleoside conjugate having sialyltransferase inhibiting activity," Cancer Research, vol. 46, no. 2, pp. 858-862, 1986.

[60] Y. Tabuchi, H. Deguchi, K. Imanishi, and Y. Saitoh, "Immunohistochemical studies on the main entrance-route of CA19-9 into the peripheral venous blood of gastric cancer patients. Correlation with CA19-9 levels in peripheral and portal blood," Cancer, vol. 66, no. 7, pp. 1529-1533, 1990.
[61] D. Marrelli, E. Pinto, A. De Stefano, M. Farnetani, L. Garosi, and F. Roviello, "Clinical utility of CEA, CA 19-9, and CA 72-4 in the follow-up of patients with resectable gastric cancer," American Journal of Surgery, vol. 181, no. 1, pp. 16-19, 2001. 


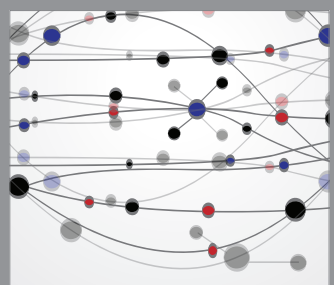

The Scientific World Journal
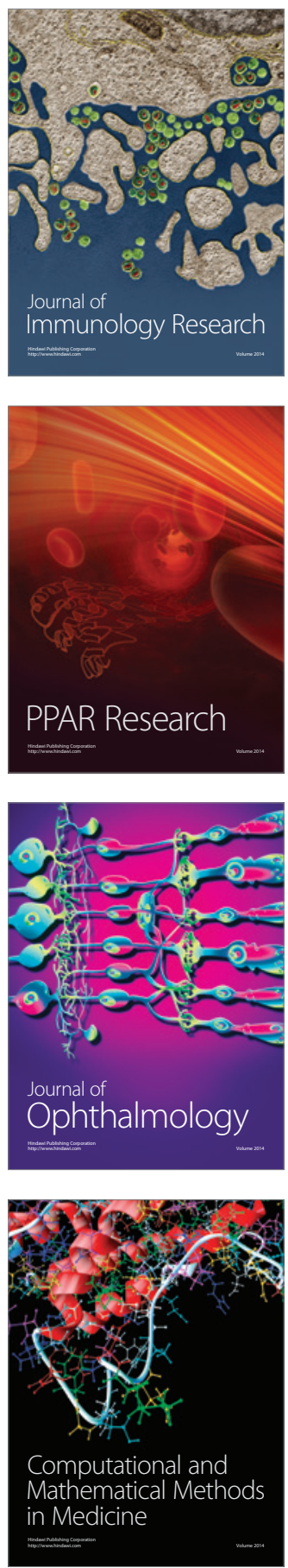

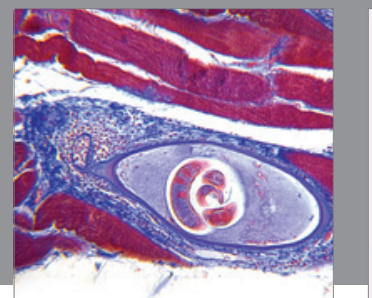

Gastroenterology

Research and Practice
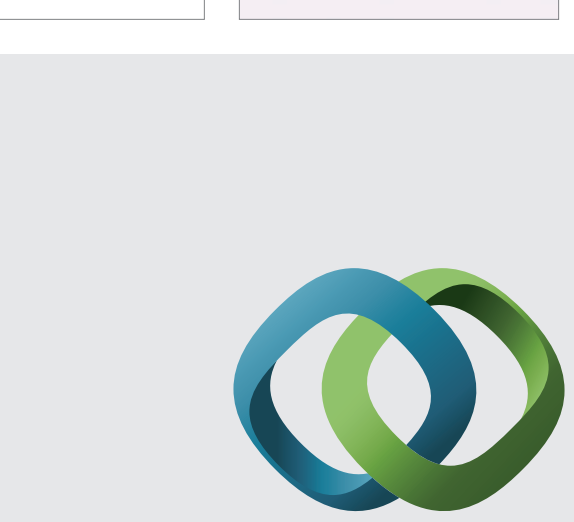

\section{Hindawi}

Submit your manuscripts at

http://www.hindawi.com
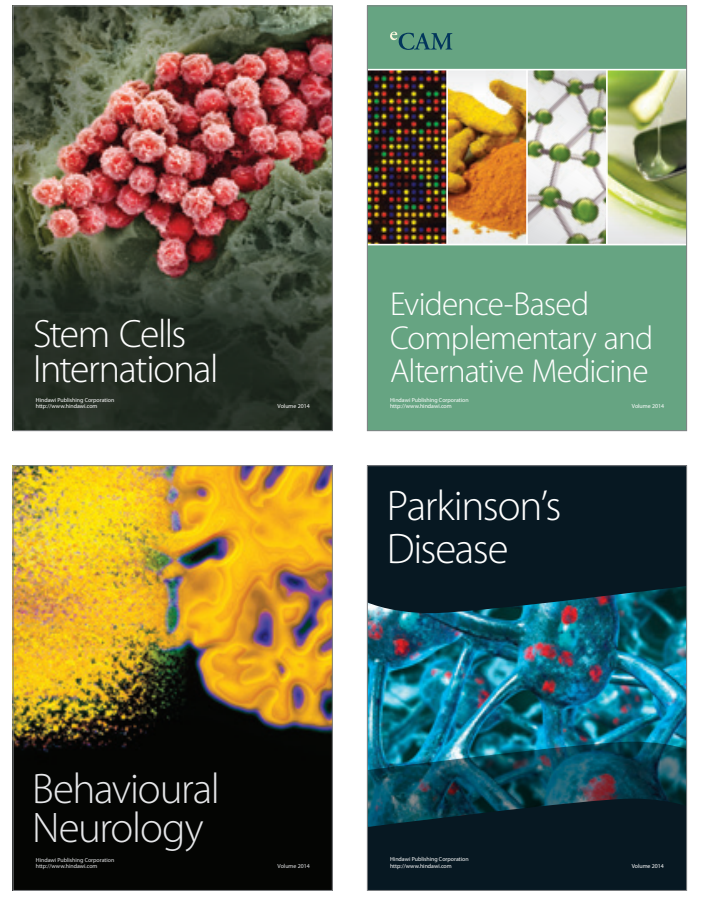
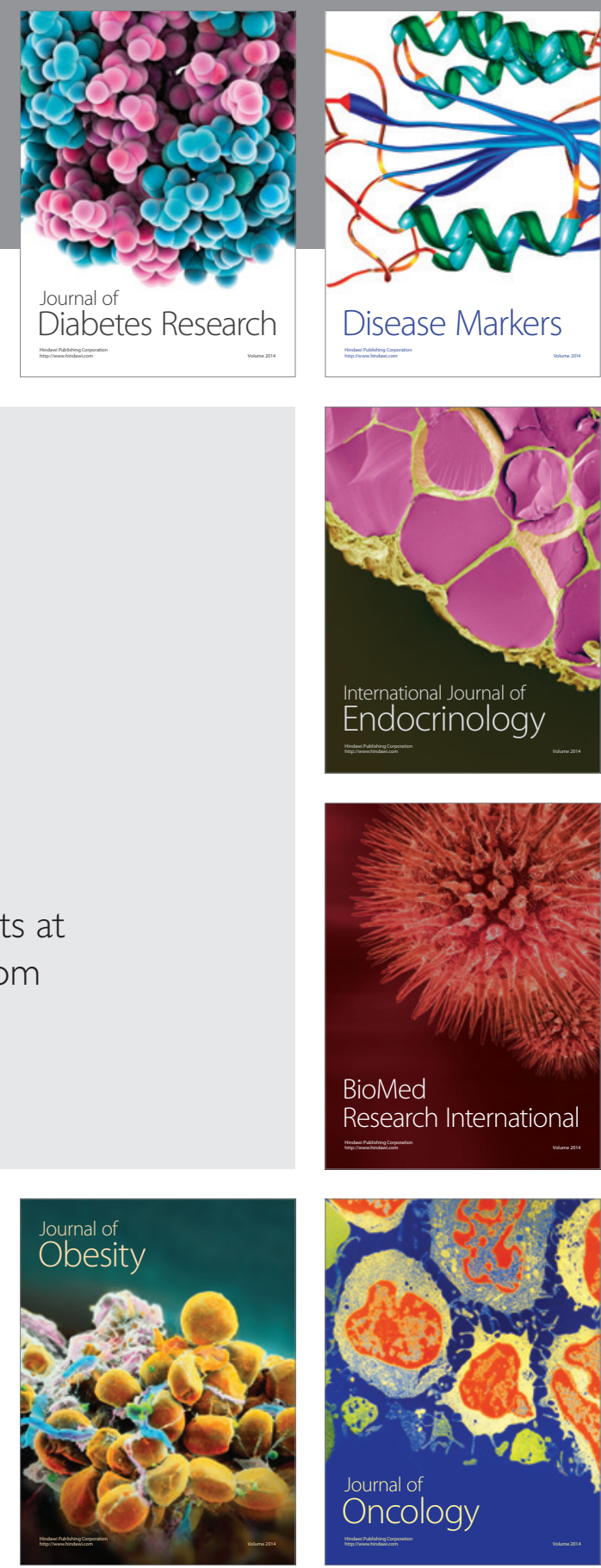

Disease Markers
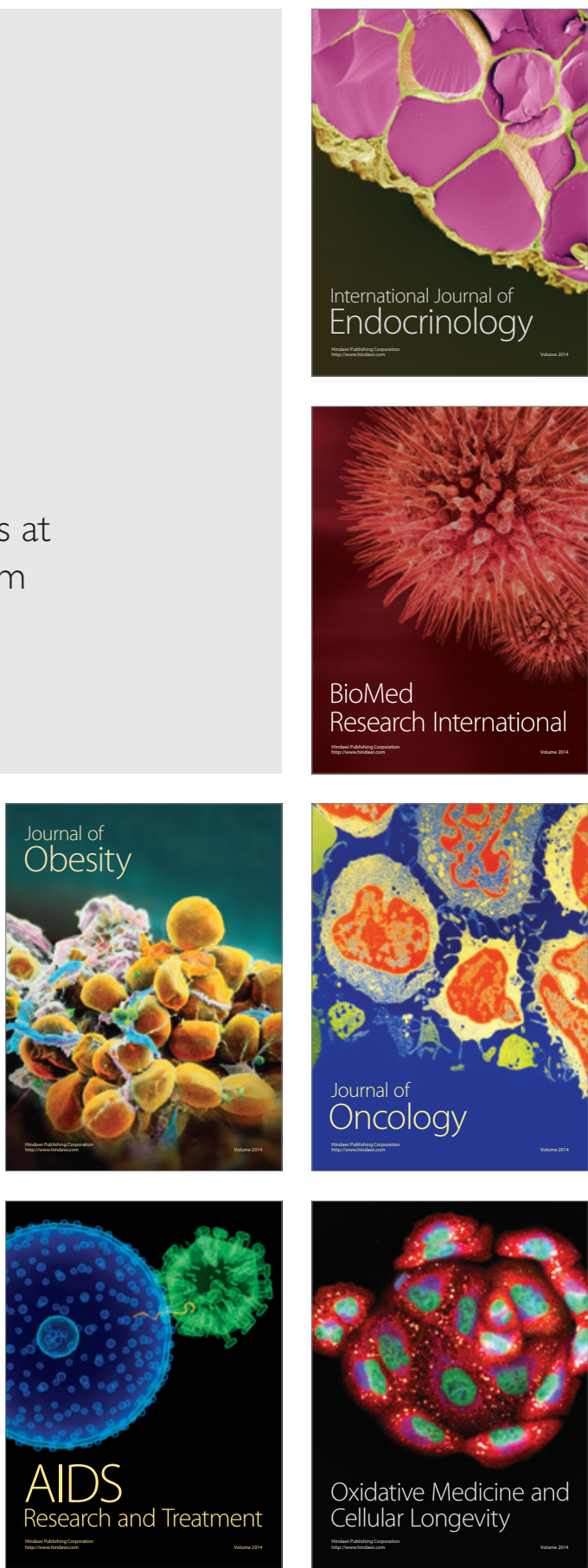